TITRE: REPRÉSENTATIONS DU DISCOURS PSYCHOTIQUE DANS L'AVANT-GARDE LITTÉRAIRE FRANÇAISE DES ANNÉES 1970

Auteur(s): JulietTe Drigny, Doctorante, UniVERsité PARIS-SORBOnNE

PUBLICATION: ÉCRITS HORS-NORMES

PAGES: $154-171$

DiRecteurs: Agnès Steuckardt et Karine Collette

ÉdITEUR: LES ÉDITIONS DE L'UNIVERSITÉ DE SHERBROOKE, 2019

ISBN: 978-2-7622-0360-8

URI: HTTP://HDL.HANDLE.NET/11143/15579

DOI: HTTPS://DOI.ORG/10.17118/11143/15579 


\section{Représentations du discours psychotique dans l'avant-garde littéraire française des années 1970}

Juliette Drigny, Doctorante Université Paris-Sorbonne

Résumé : Cet article entend aborder la place des discours des psychotiques dans le mouvement d'avantgarde littéraire français des années 1970 représenté essentiellement par les revues Tel Quel, Change et TXT, dans une perspective d'histoire des représentations linguistiques. À la faveur des réflexions théoriques contemporaines sur les rapports entre langage et idéologie, qui justifient l'intérêt pour les discours horsnorme, et de l'influence croissante de l'anti-psychiatrie, les discours des « fous », jusque-là cantonnés au domaine psychiatrique, acquièrent une place dans la sphère littéraire. Par « représentations », nous entendons d'une part la présence concrète, dans les revues, des discours de psychotiques, et d'autre part les tentatives de description qui en sont faites et qui convoquent un certain nombre de topiques linguistiques. II ressort de notre analyse que le discours « fou », malgré les parallèles établis avec la littérature, autre discours « hors-normes », sert essentiellement à mettre en valeur la sphère de la créativité littéraire : l'imaginaire littéraire des années 1970 distingue un hors-normes littéraire acceptable d'un hors-normes psychotique pur. Une brève étude des analyses contemporaines de l'œuvre autobiographique de Louis Wolfson, Le Schizo et les langues (1970), confirme cette idée.

Mots-clés : psychotiques, avant-garde, littérature et folie, Louis Wolfson, revues littéraires, post-structuralisme.

Abstract: This articles aims to tackle the place of psychotics' discourse in the French literary avant-garde movement of the 1970s, as represented mainly by the journals Tel Quel, Change, and TXT, in a perspective of history of linguistic representations. At that time, in the wake of contemporary theoretical reflexions on the relationships between language and ideology (which justify the interest taken in nonstandard discourse) and of the growing influence of anti-psychiatry, "madmen's" discourse, which was until then confined to the psychiatric realm, entered the literary sphere. By "representations", we mean on one hand the concrete presence, in journals, of psychotics' discourses, and on the other hand the attempts at describing the latter which summon a few linguistic topics. Our analysis demonstrates that "mad" discourse, notwithstanding parallels drawn with literature as another nonstandard discourse, is mainly used to highlight the creativity of literature: the literary imagination of the 1970s distinguishes between an acceptable literary nonstandard and a purely psychotic nonstandard. A brief study of the contemporary analyses of the autobiographical work by Louis Wolfson, Le Schizo et les langues (1970), confirms that idea.

Key words : psychotic, avant-garde, literature and madness, Louis Wolfson, literary journals, post-structuralism. 
«Fou», «folle», «schizo», «psychose»... à l'heure actuelle, nous n'employons ces termes forts qu'avec des guillemets, leur préférant ceux de «maladie mentale » ou de «maladie psychique ». Ils soulignent cependant l'important renversement de perspective qui s'est déroulé depuis les années 1970. À ce moment, sous l'influence de l'anti-psychiatrie, la «maladie mentale » est une notion très contestée, tandis que la folie et les adjectifs qui en dérivent sont utilisés sans connotation péjorative. Le « schizophrène », ou «schizo » tout court, acquiert alors ses lettres de noblesse.

Les années 1970, en France, sont celles de "l'avant-garde théorique », avant-garde littéraire marquée par les sciences sociales les plus à la pointe (en premier lieu la psychanalyse et la linguistique), surtout représentée dans des revues littéraires comme Tel Quel ou Change. Le « fou » envahit la littérature, et en particulier le domaine de l'avant-garde. Cette invasion de la folie est-elle si étonnante ? La présence des discours «fous » dans l'avant-garde littéraire des années 1970 pourrait n'être qu'une déclinaison d'un topos séculaire, celui de la proximité entre inspiration et délire, mania poétique; cette proximité serait même un principe fondamental si on en croit André Breton, qui écrit, à propos d'Antonin Artaud, en 1959: «Posons d'abord en axiome que la poésie, à partir d'un certain niveau, se moque absolument de la santé mentale du poète. Son plus haut privilège est d'étendre son empire bien au-delà des bornes fixées par la raison humaine. » (Breton, 1959: 981). La littérature subsumerait la frontière même entre création et folie. L'avantgarde des années 1970 échappe-t-elle à ce lieu commun? Ses ambitions scientifiques et notamment linguistiques, ouvrant un espace à l'analyse des discours hors-normes, pourrait le faire penser. Ma perspective sera donc celle d'une histoire des représentations linguistiques. Il ne s'agira pas tant de décrire le discours hors-normes des malades mentaux à proprement parler qu'envisager comment il a pu être perçu et analysé dans les années 1970, à l'époque de la «mode» de la folie.

La proximité linguistique soulignée entre discours des fous et création littéraire semble paradoxalement correspondre à une réaffirmation de la frontière entre norme et hors-norme. Le positionnement des écrivains permet de mettre en évidence une répartition entre «vraie » folie et une «sur-folie », nettement plus valorisante : celle des écrivains. Après avoir contextualisé la présence théorique du « hors-normes » dans l'avant-garde, j'envisagerai la place et la représentation des discours psychotiques eux-mêmes, avant d'observer un exemple précis, celui de l'ouvrage de Louis Wolfson, Le Schizo et les langues.

\section{Contextualisation : l'importance du « hors-norme » dans l'avant-garde}

\section{L'avant-garde post-structuraliste}

L'avant-garde littéraire qui a le plus de visibilité dans le champ littéraire des années 1970 se situe dans le courant du «post-structuralisme » (ou « ultra-structuralisme » dans l'acception de François Dosse) : il s'agit d'un second tournant du structuralisme, qui s'étend à d'autres modèles que celui de la linguistique structurale, et s'ouvre plus largement à la sphère de la créativité littéraire (Dosse, 1992 ; Kaufmann, 2011 : 18). Parfois appelée « avant-garde théorique ", très marquée par le marxisme, cette avant-garde mélange textes théoriques et textes littéraires : la pratique y est indissociable de la théorie. Ce courant présente une forte dimension expérimentale, comme l'Oulipo à la même époque, mais l'ambition d'analyse y est très forte et les créations littéraires s'accompagnent d'une abondante production théorique. 
Deux revues littéraires, en particulier, incarnent ce courant, et se partagent la visibilité dans le champ de l'avant-garde instituée: Tel Quel et Change, revues concurrentes, toutes deux publiées par les éditions du Seuil. À côté de ces revues parisiennes, gravitent quelques revues «satellites », comme Mantéia, Action poétique, ou TXT.

\section{Le hors-norme comme impératif de subversion}

Comme le formulent plusieurs titres de Philippe Sollers (une conférence de 1965, Le Roman et l'expérience des limites, puis un ouvrage intitulé L'Ecriture en l'expérience des limites en 1968 (Sollers 1968b)), l'intérêt théorique et la production artistique vont être polarisés vers les « expériences des limites » et leurs différentes variantes, textes ou langages des « limites », de la « rupture », les « grands irréguliers » du langage.

C'est une époque où se développe une conscience aiguë de l'uniformisation exercée par la langue, héritée des travaux d'Althusser qui pose le lien entre langue et idéologie (Althusser, 1970), prolongés par ceux de Balibar (1974a et b). La langue, conçue comme une superstructure, jouerait un rôle dans l'idéologie. L'idée que le langage influe sur le réel traverse nos revues : un manifeste de Change proclame par exemple que « La langue, en se changeant, change les choses. Accompagnant tous les autres gestes matériels des hommes » (Faye, 1975 : 7). Dans Tel Quel, « [...] Que le langage soit directement et immédiatement lié aux phénomènes de classe et aux changements économiques et sociaux ne saurait être contesté, même par un non-marxiste » (Delahaye, 73). Ainsi, l'impératif de subversion politique que l'avant-garde exprime doit passer par la langue: selon Kristeva, «il ne s'agit pas d'équivalence, mais d'identité entre la contestation du code linguistique officiel et la contestation de la loi officielle». (Kristeva, 1978: 83) Le langage poétique peut ainsi être perçu comme «illégalité » (Houdebine, 273). Cela s'accompagne de tentatives langagières qui se dirigent contre la norme linguistique, d'où « l'illisibilité » des textes, censée être une forme de résistance à l'idéologie.

L'illisibilité serait donc la qualité particulière d'un texte en regard de l'idéologie quant à lui aveugle. [...] L'illisibilité devient alors le point fort de la lecture, l'obstacle que celle-ci doit vaincre, la surface résistante sur laquelle vient buter et se manifester la force inerte de l'idéologie, ce qu'une société éparse en chaque individu ne doit pas lire, ne peut pas lire. [...] Ce texte est d'autant plus irrecevable par la société, par chaque lecteur en lequel vit inconsciemment les codes de langage qui assurent la domination idéologique d'une classe, qu'il désigne l'interdit et montre les limites sans lesquelles le code ne pourrait plus fonctionner. (Baudry, $1968: 140)$

La subversion de la langue s'accompagne de la recherche de modèles qui s'écartent de la linguistique institutionnalisée. Il convient de rappeler qu'en raison de la grande popularité du structuralisme, la linguistique se voit largement réappropriée par des non-spécialistes, des non-savants. Or à partir de 1966-1967 en particulier, les intellectuels prennent de la distance avec la linguistique saussurienne, qui a fondé le structuralisme. On reproche par exemple à Saussure d'avoir exclu de son champ d'étude la parole, privilégiant la langue. Sous l'influence de la récente linguistique de l'énonciation de Benveniste, se développe une préoccupation pour les sociolectes, jargons, ou langues « déviantes». D’autres concepts centraux de la linguistique saussurienne sont également remis en question, comme celui de linéarité du langage, ce qui donne lieu à l'intérêt pour les « monstres de langage » que sont les lapsus, ou les mots-valises. La publication des anagrammes de Saussure, la « part sombre » de l'œuvre du linguiste à la fin des années 1960 (Starobinski, 1964, 1967, 1971), tombe à point nommé. C'est également l'époque d'émergence, en 
France, de la linguistique générative de Noam Chomsky, autre modèle adopté à l'époque pour renouveler la linguistique et introduit en France notamment par Nicolas Ruwet à partir de 1967 (Ruwet, 1967 ; sur ce sujet, voir Dosse, 1992: 13-29).

Dans ce cadre, de quoi témoigne l'intérêt pour les discours des fous? Dans les années 1970, avec l'essor de l'anti-psychiatrie, le fou incarne pour ainsi dire la subversion ; il est en lui-même un rejet de la loi. Mais cet intérêt culturel croise également l'intérêt linguistique, dans la mesure le langage des psychotiques s'écarte de ce que l'on considère comme « normes » linguistiques. C'est cet aspect qui intéresse particulièrement nos revues.

\section{Représentations du discours psychotique}

\section{Présence du discours psychotique dans les revues}

Sollers, dans le «programme » formulé dans Logiques et repris plusieurs fois ailleurs, désigne les textes de la rupture : «mystique, érotisme, folie, littérature, inconscient» (Sollers, 1968a: 10). La folie fait ainsi partie pour Sollers des «textes de la rupture». On cherche à trouver ce en quoi le discours du fou peut instruire la création littéraire.

La perspective est différente du mouvement de l'art brut, légèrement antérieur. L'art brut, terme inventé par le Français Jean Dubuffet en 1945, rassemble en effet des œuvres de marginaux exempts de toute culture artistiques. Dubuffet, cherchant à " protéger » les créateurs du pouvoir destructeur du marché de l'art, collecte les productions et les expose dans des circuits différents de l'art officiel. C'est donc une pratique de collecte, d'anthologie, qui a besoin d'intercesseurs, et qui possède de surcroît une dimension essentiellement plastique. À l'inverse, les grands « modèles » de folie littéraire dans l'avant-garde sont avant tout des écrivains reconnus par l'institution et qui ont fait partie de leur vivant, au moins par intermittences, du circuit de l'édition officielle, qu'il s'agisse de Raymond Roussel, d'Antonin Artaud ou de Friedrich Hölderlin. L'avant-garde s'intéresse peu à la possibilité de constituer un document psychiatrique en objet artistique, mais mesure les croisements entre un ethos littéraire et un délire, une maladie mentale.

Néanmoins, les revues qui nous intéressent ont toutes évoqué ou publié des écrits de "fous », suivant sans doute un effet de mode mais reflétant aussi l'intérêt pour le hors-normes. Je ne m'attarde pas ici sur les textes d'auteurs ayant connu une pathologie mentale mais que l'institution littéraire a reconnu comme grands écrivains (Antonin Artaud, Friedrich Hölderlin...), ou de « fous littéraires » plus connus (Jean-Pierre Brisset, Raymond Roussel, Vélimir Khlebnikov) - l'intérêt pour les « fous littéraires » est une passion typiquement française, depuis Nodier jusqu'aux recherches actuelles de Marc Decimo, en passant par Raymond Queneau et le Collège de Pataphysique - , mais je parlerai plus spécifiquement de textes qui relèvent davantage de l'ordre du document inédit. Voici quelques exemples dans les différentes revues.

Dans Tel Quel, sont publiés des textes de Sophie Podolski, jeune artiste schizophrène morte à vingt et un ans. La publication de ses textes et dessins en fac-similé dans la revue révèle l'importance donnée à la graphie (Podolski, 1973a et b et 1977). 
Jean-Jacques Abrahams, plus connu sous le nom de «l'homme au magnétophone », est assez célèbre pour la controverse qu'il a déclenchée entre Sartre et Pontalis, à propos de la psychanalyse (Sartre, 1969). Abrahams apparaît dans trois numéros de Tel Quel.

Je vais écrire dans le nouveau TQ un article qu'ils vont accepter concernant la voix concernant Marie-Claire (Boons) et Pierre (Guyotat) et tous retrouvés dans la fraternité et je vais dire qu'ils sont là et qu'ils ont rempli le regard de Yahweh de tout ce qui a été dit à propos de Yahweh et que dès lors ils ont été sauvés et que dès lors il ne faut pas les déclarer devant être déclarés autre chose que ce qu'ils sont dès lors ce n'était le moment provisoire [...] (Abrahams, 1976 : 102-103)

Change laisse également entendre et lire des documents véritablement « psychiatriques ». Dans le numéro 32-33, tout entier consacré au thème de la folie ( La folie encerclée »), deux documents (une lettre anonyme à Jean-Pierre Faye et un enregistrement effectué à l'hôpital psychiatrique de Maison blanche) sont retranscrits. De même on trouve, dans les actes du colloque « Change » en 1975, une retranscription d'une séance du Grand Groupe (Sabourin, 1975), un groupe de parole de la clinique de La Borde, clinique fondée par le Dr Jean Oury, pionnière dans le domaine de la psychothérapie institutionnelle. Dans cette prise de parole de « Jacques », on observe une décomposition du mot « télévision » en éléments à la fois phonétiques et alphabétiques:

Eh bien T, T, E accent aigu, règle à dessin, T-é et L-é, c'est quoi pour vous ? Oui Lait : L.A.I.T., pt'être bien, mais enfin si vous voyez L.-é., L.A.I.T, vous êtes un pécore, pas le L.A.I.T. qui faut r'garder, pécore, pécore, Yvette vous êtes une pécore. [... .] Alors si vous voulez pas voir Lait, comme le lait qu’on boit, comment écrivez-vous-L-é, comment vous l'écrivez : Télévision, comment vous l'écrivez ? [...] L- E accent aigu, j'ai dit très fort, et non L.A.I.T. qui faut r'garder, pécore, pécore, Yvette vous êtes une pécore. [...] Alors si vous voulez pas voir Lait, comme le lait qu'on boit, comment écrivez-vous-L-é, comment vous l'écrivez: Télévision, comment vous l'écrivez ? [...] L- E accent aigu, j’ai dit très fort, et non L.A.I.T. [...] Eh bien, si vous voyez pas VU, qu'est-ce que vous voyez, vous voyez VISION. Alors on dit TE-LE-VISION. Si vous voyez pas VU, si vous voyez que V.I. vous dites V.I. et bien Victoire Italienne, je dirai, et V.I. - V.ZI-O.N., ZION, une des collines de Jérusalem.

Quant à TXT, elle choisit par exemple Jules Doudin, repéré par le spécialiste d’art brut Michel Thévoz, dans son numéro 10, l'ÉCRIt, le CacA. Un peu en décalage par rapport aux deux autres revues, TXT s'intéresse, plus généralement, à toutes les formes de « déviance » dans le discours, et son intérêt sur la folie se perçoit davantage dans la publication de «fous littéraires » comme Brisset ou Khlebnikov. La posture y est moins surplombante et plus intégrante. Jules Doudin a recours à la métagraphe (ou néo-graphie), c'est-à-dire une altération de l'orthographe qui n'attente pas à la forme phonétique des mots. Quand on dépouille le texte de toutes les altérations orthographiques, il en reste des histoires scatologiques ou graveleuses.

Mat merdre nat pas repouzcer qua greandes

Peaines Mont cuptzs ge les prends atdetbeout ge l'eai Vuzs zur les chemptzs de batteaille, ge vous dis ques ca. Prenez zeoin de monz cus et mas zizzolle, ge vous ferraz cadeaux des monts cups, qui et ans Décomppotzision as la 
maizon est l'oeil anst verres ques jeai eut pour

mes regarder la Guelettent mas feai renêtrent

jour pour jour dans le cheamp out geai

Vut le geardes cheampêtres, mont cuptz il est

pretzs à vous azeaizonnez quand mat merdes

reantreaz ge vous ferez caddeaux de mont

Cuptzs (Thevoz, 1978: 15)

\section{Des tentatives de description}

Je m'interrogerai à présent sur les analyses qui accompagnent ces documents. Pour caractériser le discours fou, la description hésite généralement entre ce qui relève de l'atteinte au lexique (néologie, orthographe), de l'atteinte générale au sens, ou de l'atteinte à la syntaxe. On relève quand même différents invariants, différents lieux communs qui reviennent dans la description.

La prédominance donnée au signifiant sur le signifié serait le premier attribut du discours psychotique. Par opposition au signifié, le sens donné au mot, le signifiant désigne la matérialité du langage, qu'elle soit phonétique ou graphique. Le discours psychotique aurait pour caractéristique d'insister tout particulièrement sur la matérialité du mot : les psychotiques s'attardent sur les sonorités, sur les lettres, sur la graphie. D'où l'intérêt de publier des fac-similés, par exemple pour Sophie Podolski. C'est ce que l'on a pu observer également dans la retranscription des paroles de Jacques au sein du grand groupe, dans le colloque «Change». De même, le texte psychotique présenterait des mécanismes de création par « jeux sur le signifiant », c'est-à-dire de génération du texte par des effets phonétiques, glissements ou paronomases. Jean-Pierre Brisset ou Raymond Roussel forment d'ailleurs, en ce domaine, des cas d'école ; dans sa Grammaire logique de 1883, "résolvant toutes les difficultés et faisant connaître par l'analyse de la parole la formation des langues et celle du genre humain », Brisset entend prouver, par des procédés d'étymologie fantaisiste, que l'homme descend de la grenouille. Quant à Roussel, dans Commentj'ai écrit certains de mes livres, il explique que l'intégralité d'Impressions d'Afrique a été écrit selon un procédé inventé par lui : « Les lettres du blanc sur les bandes de billard» (signes typographiques écrits à la craie sur les bordures) sont ainsi devenus « les lettres du blanc sur les bandes de pillard » (lettres écrites par un homme blanc à propos de hordes d'indigènes).

La prédominance du signifiant amène à une seconde représentation, celle des «mots-choses », qui en est en quelque sorte une déclinaison. La folie introduirait, dans la langue, un rapport concret, palpable, aux mots. "Il faut dire que dans le langage psychotique les mots ne constituent plus un système de représentation des choses, mais qu'ils sont eux-mêmes des choses. » (Sabourin, 1975 : 64). Davantage, les mots du psychotique seraient perçus comme un prolongement du corps du locuteur. On parle ainsi de « corps dans la langue », de «langage du corps », voire de « corps langage »... On trouve ainsi ce type de formulations : «Le mot est partie du corps » (Sabourin, 1975 : 103) ; « il s'agit ici d'un corps langage et non d'un langage du corps » (Sabourin, 1975 : 65). «Il nous semble que l'organisation psychotique, dans sa différence avec les organisations "normales" ou névrotiques, se caractérise par le défaut de clivage, ou d'articulation, assuré entre l'espace littéral et le corps. La lettre ne cesse de se rabattre sur le corps et rien ne permet alors de distinguer le corps des mots. » (Leclaire, 1972: 126). 
Les mots-choses seraient ainsi dus à la négation des frontières, troisième topique que nous allons envisager. Ce thème est présent à différents niveaux : la négation des frontières du mot lui-même produit le morcellement qu'on observe dans les mots disloqués, les syllabes et les lettres disjointes. À cela s'ajoute la négation des frontières syntaxiques, qui peut produire par exemple les phrases sans bornes de JeanJacques Abrahams. Le psychotique serait aussi à même de nier les frontières entre les différents idiomes, dans les cas de Brisset ou de Wolfson.

Enfin, ces discours psychotiques donnent lieu à quelques tentatives de description syntaxiques, notamment selon la grammaire chomskyenne. Le n`16-17 de Change (1973), consacré à «la critique générative », propose ainsi une tentative d'analyse « dendritique » d'une phrase de schizophrène. L'approche par « dendrite » est une reformulation de la formalisation chomskyenne par les arbres, le mot « dendrite » provenant initialement de la biologie (ramifications neuronales) et ou des fractales.

Le langage du schizophrène est un langage dendritique, dont chaque terme renvoie à de multiples sens. Si le langage de la norme se veut linéaire, implanté dans un monde univoque, essentialiste, où toute association non pertinente au message se trouve sévèrement refoulée, le schizophrène, lui, joue constamment avec les mots. Les associations se multiplient [...], détruisent non seulement le sens, mais jusqu'à la syntaxe du message. (Moreau-Hicks, 1973 : 128)

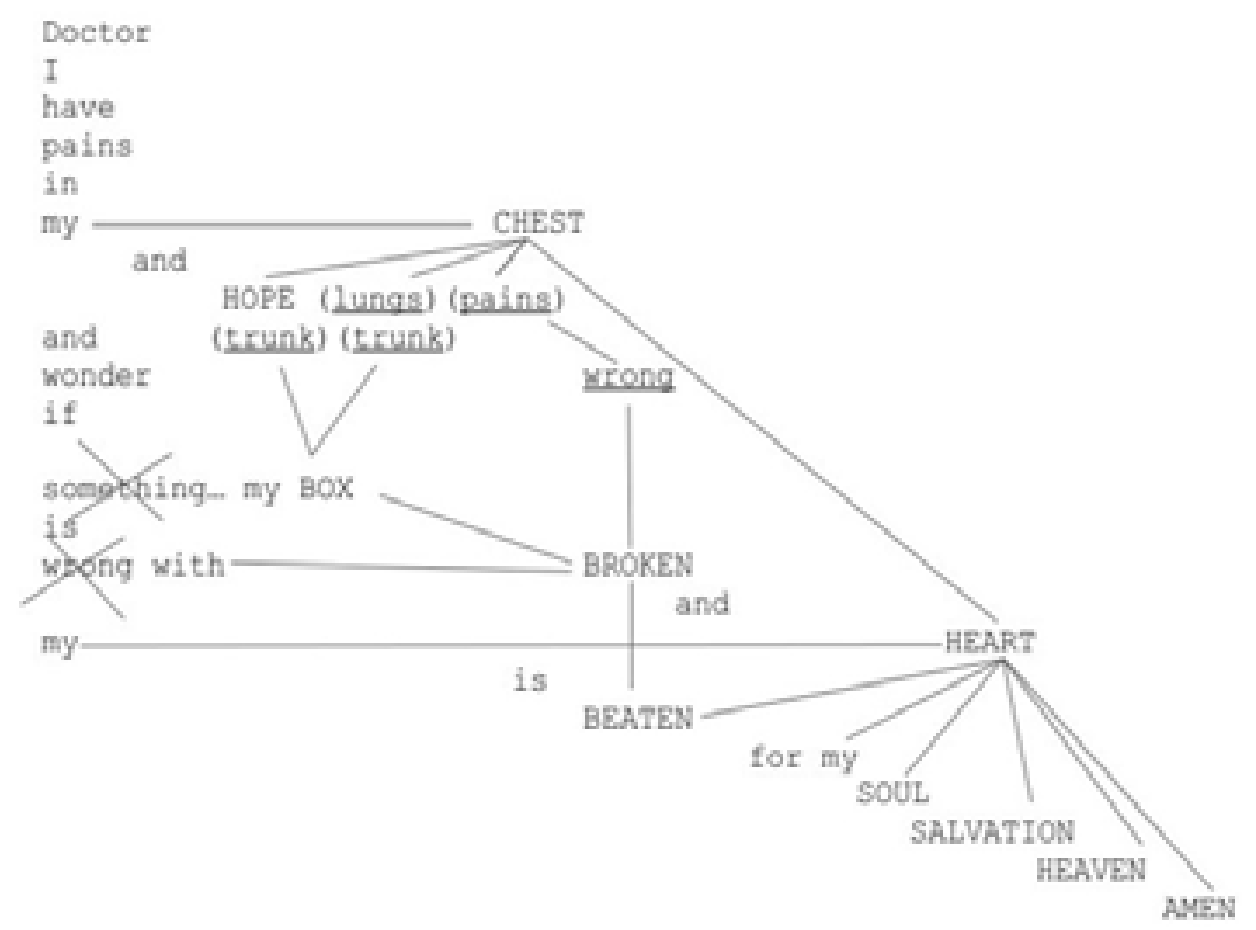

Figure 1. Moreau-Hicks (Change, 16-17, $1973:$ 128)

À partir de cette phrase, "Doctor, I have pains in my chest and hope and wonder if my box is broken and my heart is eaten for my soul and salvation and heaven, amen ", l'auteure propose un graphique censé rendre compte de l'ambiguïté syntaxique et des incertitudes. Cette démarche est assez intéressante, puisqu'elle se fonde en réalité sur quelque chose qui n'est pas actualisé dans la grammaire générative de Chomsky : la « créativité » se tient dans les strictes limites de la grammaire mentale, ne laissant ainsi que très peu 
de place à l'ambiguïté (Joseph, 2011 : 4-7). La formalisation du discours des psychotiques est un premier pas, dans Change, vers la grammaticalisation du discours littéraire lui-même. C'est toute l'ambition de ce numéro sur « La critique générative »; Mitsou Ronat, par exemple, va proposer des « règles » de traduction de Mallarmé, en s'exerçant sur un texte en anglais du poète (Ronat,1976a, b et c).

En réalité, ces différentes tentatives de description reflètent assez bien l'hésitation généralisée concernant l'analyse des discours des malades mentaux. Les discussions suivant l'intervention de Sabourin et Polack au colloque «Change » (Sabourin, 1975) révèlent ce changement permanent de niveaux d'analyse, entre lexique (Jacques Roubaud n'y voit aucune rupture avec les règles linguistiques, mais de simples jeux sur le lexique), syntaxe, pragmatique et énonciation (pour Danielle Sabourin, c'est au niveau du langage comme comportement social qu'il y a des ruptures). On peut de même observer, dans l'intervention suivante de Michel Armelino, un glissement progressif depuis un plan linguistique jusqu'à un plan pour ainsi dire existentiel :

Aujourd'hui, j'essaye de comprendre ce qui se dit, un peu comme s'il s'agissait de quelqu'un qui a même instrument, même langue que moi, mais qui, d'une certaine manière, bouscule la syntaxe, combine les mots entre eux d'une manière totalement différente, totalement subversive, qui effectue une sorte de voyage, qui a une expérience de la réalité complètement différente de la mienne. Cependant à essayer de cerner cette réalité du fou, on risque de glisser à l'intérieur de cet univers [.. . ]. Une question se pose par rapport à ce discours, celle de la vérité. (Armelino, 1975 : 25)

C'est le développement des questions linguistiques vers une dimension existentielle qui explique, notamment, les parallèles établis avec la littérature.

\section{Le lien établi avec la littérature}

Tout d'abord, la folie semble interroger le processus de création littéraire lui-même. Ce point de vue est commun dans les deux revues rivales.

Le processus en activité, les débuts schizophréniques, sont des moments de grand remaniement. [...]. Tout créateur authentique s'y reconnaîtrait à telle ou telle phase de son évolution. C'est le moment où les déchirements, les fendillements, les béances, apparaissent et laissent apparaître un autre monde, en soi, insoupçonné. (Zoïla, 1975 : 153)

Dans Tel Quel, Daniel Sibony livre un long texte, «Écriture et folie», sur cette association.

J'ai dit que l'écriture littéraire poétique qui n'a rien à voir avec la folie est pourtant ce qui l'approche au plus près et s'en différencie à l'extrême. (Sibony, 1977: 48)

Chez Julia Kristeva et Mitsou Ronat, les deux linguistes des revues concurrentes Change et Tel Quel, ce sont sur la syntaxe que les comparaisons portent.

En schizophrénie et dans le langage poétique du texte moderne, la négation et la structure syntaxique trouvent leur statut transformé ou leur normativité perturbée [...]. La négativité stoppée-absorbée dans la négation du jugement, ne transparaît donc qu'à travers des modifications de la fonction 
de la négation ou dans des modifications syntaxiques et lexicales, propres au discours fou ou à la poésie. (Kristeva, 1974 : 114)

Le lien du désir, de la langue, et des formes poétiques est loin d'être clair, bien que soupçonnable, intuitivement. Ne voit-on pas le caractère obsessionnel du décompte des syllabes dans le vers, la problématique de la répétition, l'utilisation schizoïde de règles grammaticales existantes ou, perverse, des imbrications? (Ronat, 1975 : 175)

Davantage, on peut se demander s'il n'y a pas eu une influence des représentations du discours psychotique sur le style littéraire lui-même, dans les productions littéraires de l’avant-garde.

On peut trouver à mon sens observer que les textes « littéraires » reproduisent un certain nombre de topos à propos des discours «fous » que nous avons identifiés ci-dessus. Le style propre aux textes de Tel Quel, ainsi, est riche en « jeux sur le signifiant » et paronomases. Voici à titre d'exemple un passage de « l'état Artaud » de Sollers, « calque » d’Artaud, évoquant sans Pour en finir avec le jugement de dieu.

\section{Qu'est-ce qu'un gli?}

Le papier graisseux, le coton, l'emballage, le fond blanc de culotte en trou noir opposé au cri. Non pas au cri crié, immédiat, par exemple celui du cochon qu'on égorge, de l'épileptique dans une salle de cinéma, ou encore celui, plus naissant, de telle ou telle hystérique attrapé au vol au moment où le tranchant siffle, mais cet autre cri, autrement étalé et désintriqué, autrement tassé dans son autre, qu'un certain usage de la dépense et du geste sorti en lui-même, autrement strié, finit parvous enlever. C'est alors que les choses penchent et, en fait, se dressent. C'est alors que le gli s'installe pour les redresser.

C'est finalement l'accusation qu'il porte, qu'il ne cesse pas et ne cessera pas de porter et il ne s'agit pas du tout d'un certain légendaire ou fabuleux manque à être où l'essentiel en soi perdrait son essence en cours de route avant d'arriver à celui qui l'énoncerait, non, mais bel et bien d'un meurtre en bonne et due forme ou informe accompli, perpétré, calculé consciemment et surtout inconsciemment par un pli qui s'est pris dans l'humain pour pli, qui s'est épris de son repli, qui n'arrête pas de sucer, avec ou sans visage, son église en gli.

Qui intronise le gli, l'émet, l'émane, le diffuse et le reproduit? [...] C'est le gli qui se prend pour cri. Qui voudrait être gli dans le cri pour pouvoir commenter le cri. Et par conséquent en faire de l'écrit [...].

C'est comme ça, dans nos régions, qu’on fait rentrer les sorties. (Sollers, 1972: 5-6)

La « folie » de ce discours - qui tourne autour d'un néologisme non défini ! - est mêlé d'effets de rhétorique et d'allusions précises au texte d'Artaud. La « folie » du texte devient un moyen de créer une certaine obscurité, inscrite dans un système rhétorique d'allusions servant à créer un sentiment de connivence chez le lecteur averti.

Dans un texte d'Yves Buin (figure 2), les lettres sont traitées comme des objets d'une façon métalinguistique. 
Prenons M transfusion cerveau Renversons W écoute même si tu flottes nous obtenons bain de mère compris Alors maintenant $\mathrm{AB} /$ professeurs de Munich guident le fluide/Ca donne $\mathrm{ABT} T$ vous savez bien pas la peine de raconter Les professeurs ne connaissaient pas ABT m'ont envoyé ici pour ça Je répète $\mathrm{AB}$ manque $\mathrm{T} T$ je le garde on ne sait jamais je vous dirait $\mathrm{T}$ le matin et vous pensez à $\mathrm{MW} M$ rentre dans $\mathrm{W}$ pas/W dans $\mathrm{M}$ parce que $\mathrm{ABT}$ Donc $\mathrm{T}$ est à moi Surtout ne pas croire que AB tient tout seul $\mathrm{T}$ à l'envers ? Jy ai pensé ça fait BCJ J Jaime mieux $\mathrm{A}$ à l'endroit trop dangereux de le mettre à l'envers $\mathrm{V}$ se rajoute une petite barre $\forall$ pas fermer la porte s'ils voyaient ça ! Rangez vite $\forall$ car je n'ai pas le droit de l'écrire je dois m'arrêter à BCJ Je dois vous faire oublier ABT [...] J ? C'est $\mathrm{G}$ dans le miroir regardez $\mathbf{G}$ mais c'est très loin de $\mathrm{V}$ ça me brûle comme le pourpoint Vous ressemblez à ceux de Munich avec vos yeux de murs L'algèbre dit que vous m'aurez vivante $\mathrm{ABT} A \mathrm{ABJ} \mathrm{V}$ j'ai mis trente millions d'années à perdre mes mains pour la formule.

Figure 2. Yves Buin (Change, 12, 1972: 139)

Ce jeu sur les éléments alphabétiques évoque de manière évidente les « mots-choses ».

Un dernier texte publié dans TXT, «L'esprit fou dans les grands vents » de Denis Roche (Roche, 1970 : 65), fait partie d'une série intitulée « dépôts de technique et de savoir », dans laquelle D. Roche collecte et assemble des fragments de textes épars, extrêmement variés. Différents éléments peuvent nous rappeler le langage des psychotiques : la fragmentation et le non-respect des frontières syntaxiques, le caractère accumulatif du discours, et l'insistance sur la matérialité graphique (la forme du cadre). Tout en possédant une cohérence thématique globale (le vent, la sexualité), le discours est impossible à « suivre » précisément...

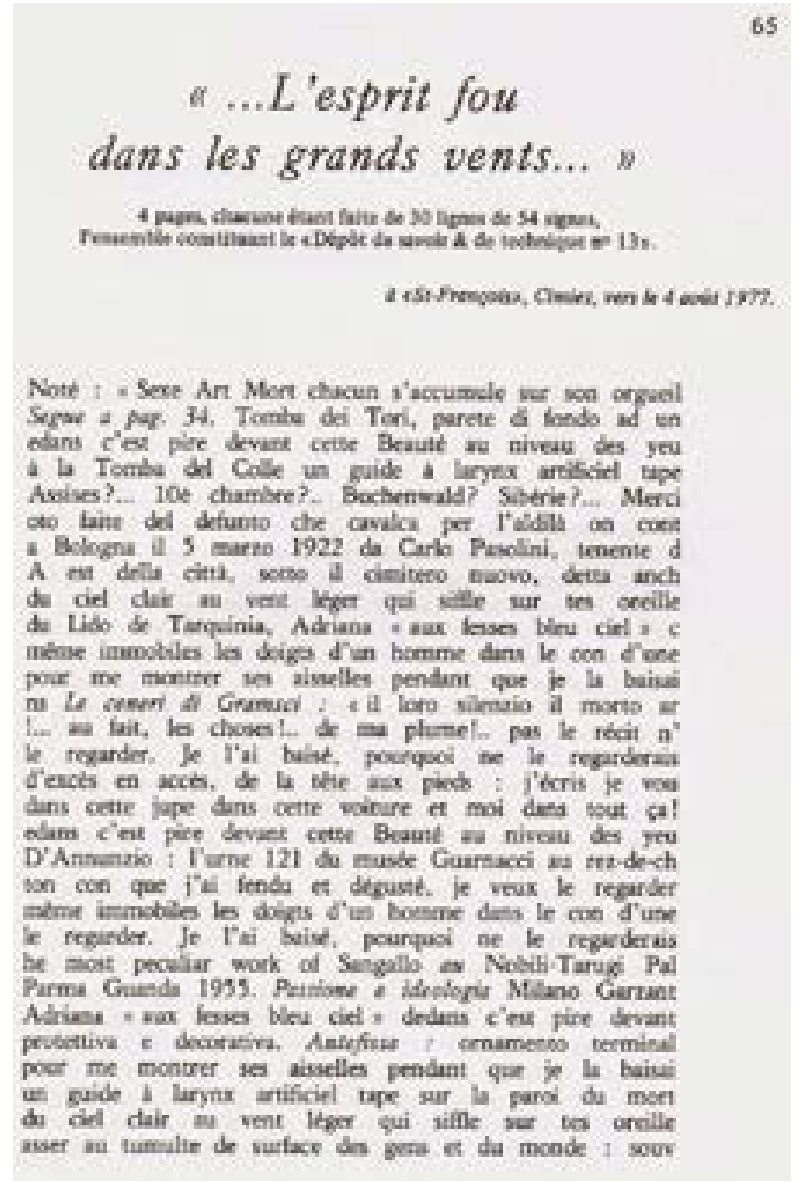

Figure 3. Denis Roche (TXT, 10, $1970: 65)$ 
Mais la folie évoquée est extrêmement « littéraire », dans ce texte encadré par Diderot, de qui provient la citation « l'esprit fou dans les grands vents », et la citation finale « Adieu, je sens que l'yvresse me gagne ». Le texte semble ainsi calquer avec distance la folie davantage qu'il ne l'incarne.

\section{La revalorisation des pouvoirs du littéraire}

Ainsi, la comparaison a ses limites : on prend bien garde à cerner le champ de la folie. La littérature est toujours « au-delà » de la folie. "Le fou aussi est suspendu à cette béance du signifiant et il la lie à ellemême, elle est son propre recours dans le maintien du monde psychotique. L'écrivain-poète, lui, a franchi quelque chose. » (Sibony, 1977: 51). La revue Change, bien qu'elle se prétende plus sérieuse que Tel Quel dans son appréhension de la folie - elle tient à marquer la distance avec « l'idéologie de la "belle psychose" » et à se « rattache[r] à l'investigation froide de la déraison » (Faye, 1974:16) - présente le même type de phraséologie : "Le récit touch[e] aux universaux et [la] narration ne p[eut] proposer de la folie qu'une quête au-delà de la "folie" » (Montel, 1972: 167). Kristeva, de même, dans la Révolution du langage poétique, prend en réalité grand soin de différencier le langage poétique et le discours du névrosé : " On pourrait dire que le texte comme pratique signifiante est l'activité de la folie, ou une folie active c'est-à-dire "socialisée " » (Kristeva, 1974 : 187). Le langage poétique, contrairement au langage des fous, aurait selon elle la capacité à définir un nouveau dispositif, il est structurant.

On observe ainsi, en réalité, une sélection dans le donné de la folie. Daniel Sibony annonce se limiter au " délire, formation plutôt "riche" de la psychose » (Sibony, 1977 : 46). De même les écrivains de Tel Quel (Sollers et Kristeva notamment) posent une différence sexuelle dans le donné de la folie : seule la folie masculine pourrait donner lieu à une atteinte au langage vraiment créatrice (Sollers, 1977b). Pleynet, dans Stanze, distingue de même ce qu'il appelle le «schizo.bête » du «schizo.intelligent » (Pleynet, 1973 : 158159).

L'acte littéraire, ainsi, est présenté comme un acte risqué qui côtoie la folie mais doit en réchapper :pour Sollers, « les deux bords du risque s'appellent débilité et psychose » (Sollers, 1977a : 8). La question de la folie fonctionne en définitive comme un moyen de revaloriser le littéraire. L'écrivain par excellence est celui qui reproduit les caractéristiques du discours fou (les jeux sur le signifiant, la rupture du sens) sans être fou lui-même. Il se produit ainsi un nouvel élitisme. Sollers écrit que "s'occuper de quelque chose qui touche le discours de la folie devrait être le fait d'une sainteté absolue » (Sollers, 1977b : 101). Une semblable exigence de maîtrise, par rapport au discours fou, s'observe dans son texte «La renommée » : le "surpayeur de renom » (c'est-à-dire le grand écrivain) «n'écrit pas n'importe quoi ».

La naïveté littéraire est désormais exposée au grand jour : elle a beau grincer des dents, exploser, coïter, déféquer, s'onomatoper, se masturber, s'enkyster, se sodomiser, se mettre au lapsus laborieux ou au calembour, crier, hurler, gesticuler, sussurer, rien n'en sort qu'un air définitivement entendu du surveillant social de service. Ici, flash d'Artaud:

«C'est que votre sperme est très bon,

m'a dit un jour

un flic du Dôme

qui se posait en connaisseur,

et quand on est «si bon », 
«si bon», dame,

on surpaye

son renom. »

[...] Contrairement à l'analysant [...], c'est-à-dire n'importe qui, vous, moi, tous les autres et la majorité des écrivains en tout cas ; contrairement à l'analysant, donc, le surpayeur de renom N'ÉCRIT PAS N'IMPORTE QUOI. (Sollers, 1975 : 101-102)

\section{L'exemple de Louis Wolfson}

Le Schizo et les langues, texte autobiographique de l'Américain Louis Wolfson publié en 1970, est écrit en français à la troisième personne. Ne pouvant supporter l'écoute de sa langue maternelle, l'anglais, et se passionnant pour l'étude des langues étrangères, Wolfson en vint à inventer un procédé pseudo-linguistique de conversion des mots anglais en des mots étrangers. Le livre a été publié chez Gallimard, dans la collection «Connaissance de l'inconscient » créée par Pontalis, une collection de théorie psychanalytique: dès sa parution, le livre a donc un statut proche du document. Il connaît toutefois un assez fort écho dans le champ de l'avant-garde: Alain Rey en fait un compte rendu dans Critique, une revue proche de Tel Quel (Rey, 1970), et des extraits remaniés paraissent dans Change (Wolfson, 1977 et 1978). Voici le début de l'ouvrage:

Le jeune homme schizophrénique était maigre comme beaucoup de gens dans de tels états mentaux. En effet il semblait plutôt dénourri. Peut-être était-il même dans un état de marasme; du moins sa mère semblait-elle parfois penser ceci. [...] Le jeune homme avait été dans beaucoup d’hôpitaux d’aliénés, presque toutes les fois son transport, sinon aussi son admission automatique, ayant été arrangé préalablement et à son insu par sa mère, comme très souvent on le fait d'une telle manière sournoise avec les gens que les psychiatres jugent ou du moins disent malades. En ce cas le diagnostic avait été la schizophrénie, terme emprunté au grec et qui veut dire étymologiquement : esprit fendu.

La troisième personne du singulier qui inaugure le récit laisse lire la description lucide d'un personnage, alors que «le jeune homme schizophrénique » parle de lui-même. Par ailleurs dans le livre il ne se nommera jamais, se désignant par des périphrases, comme «l'étudiant en langues dément », « le psychotique », «le schizo », «le jeune homme malade mentalement »..

Conséquence directe de la rédaction de l'ouvrage en français, qui n'est pas la langue natale de l'auteur, la présence de «fautes » (solécismes essentiellement, quelques barbarismes) en fait à proprement parler un discours hors-normes.

Pourquoi et comment avait-il encore une fois fait cette bêtise de manger si beaucoup ? (Wolfson, $1970: 50)$.

Le psychotique [...] ne pouvait pas s'empêcher de songer à dire quelque chose de blâme à la mère de ce trio de méchantes enfants « volantes» (comme, semblait-il, des meules de moulin) et pour que pareille conduite ne recommençât plus. (Wolfson, 1970 : 226).

Et il continue de faire cette concession en leurs relations verbales, lesquelles sont beaucoup maigres. (Wolfson, $1970: 245$ ) 
Dans l'usage des modes verbaux s'observent aussi des impropriétés, dont la première est l'usage du conditionnel comme temps du récit, dont on ne sait pas réellement s'il s'agit d'une erreur de langue due à un calque de l'anglais ou d'un symptôme psychiatrique indiquant un rapport à la réalité biaisé.

- Qu'est-ce que cela fait? Allons ! et la jeune et très jolie fille de joie s'engagerait, malgré le clignotement impérieux en rouge des mots « NE MARCHEZ PAS », dans le passage clouté où elle ne serait point seule, la plupart des gens semblant faire de même [...]. (Wolfson, 1970 : 81)

Mais ce qui frappe surtout dans le texte, c'est le procédé développé par Wolfson. II s'agit de trouver, pour chaque mot de la langue maternelle, un mot étranger de sens similaire, mais ayant toujours des sons ou des phonèmes communs. Par exemple don't trip over the wire deviendra tu'nicht trébucher über èth hé zwirn, mélange d'allemand, de français et d'hébreu. Ces développements peuvent occuper jusqu'à une quarantaine de pages pour un seul mot.

Le schizophrène réflechissait : «Where... [et cela même de manière à faire valoir chaque lettre de ce monosyllabe, donc houèer !]. Il serait bon de m’en débarrasser tout à fait de ce mot maintenant avant qu'il ne m'embête de nouveau. Where, ça serait wo (vô) en allemand; mais l'h [que sa mère n'avait même pas prononcé !] et l'r [articulé à cause de sa liaison] restent. Voyons, $h$ et $r .$. il y a l'allemand hier (hîr, h senti) mais ça veut dire ici. Bah ! ça ne semble pas bon... mais... peut-être... Where (= où) pose la question de place, de situation. Possiblement, pour parer à la question: Where? je pourrais pense vite à «Wo ? (Où ?) Hier? (Ici ?) », ce dernier comme possible réponse à la question proprement dite, l'un aussitôt après l'autre, ou plutôt je convertirai instantanément Where? en tous deux Wo ? et Hier? Mais peut-être le sens de hier est-il trop écarté. Et en certains cas, même contraire! (Wolfson, 1970 : 6768)

Si j'observe à présent la réception du texte dans les milieux de la théorie littéraire, l'on retrouve les topiques que sont la mise en avant du signifiant, les mots-choses, la dislocation : pour Deleuze, auteur d'une importante préface, «les mots ont littéralement éclaté dans leurs éléments phonétiques. » (Deleuze, 1970 : 16). De même, Judith Milner lit le texte sous le prisme de la négation des frontières, frontières externes entre les langues, mais aussi les frontières internes, car il ne dédaigne pas décomposer un mot en syllabes traitées diversement (Milner, 1977).

Une question se pose donc: est-ce de la littérature? Pour Deleuze « Le livre de Wolfson n'est pas du genre des œuvres littéraires ou œuvres d'art, et ne prétend pas l'être. » (Deleuze, 1970 : 8). Il emploie le terme de « simulacre ». Elisabeth Roudinesco avance sensiblement les mêmes idées, sans doute conditionnées par la lecture de Deleuze, dans Action poétique, "Prenant [la folie] comme seul référant de son récit, le schizo ne fait pas œuvre d'art; le procédé "empêche" le texte de se dire ; il le "devance" comme il protège le fou de sa vérité. » (Roudinesco, $1970: 63$ ).

Qu'est-ce alors que ce texte ? La quatrième de couverture l'indique : un « livre exceptionnel ». De même pour Alain Rey, «Wolfson a construit un objet admirable. Non pas, c'est vrai, une œuvre d'art, ni le récit d'une découverte. Mais un texte marqué, marquant, dont le signifiant pèse autant et plus que celui d'une quelconque “littérature”. » (Rey, 1970 : 678). Pour Julia Kristeva : « La littérature américaine, c'est peut-être Cage, c'est peut-être Bob Wilson ou, pourquoi pas, Wolfson, Le Schizo et les langues, donc quelque chose qui ouvre le verbe vers ce qui ne se parle pas, avec tous les risques de psychose que cette percée suppose. » (Kristeva, 1977 : 5). Le seul moyen d'accueillir Le Schizo dans la littérature serait d'ouvrir le concept de «lit- 
térature » à l'art non-verbal. Paul Auster, ainsi, s'il refuse qu'on considère Le Schizo comme un document, considère que Wolfson « se tient en dehors de la littérature telle que nous la connaissons » et qu'il faut lui créer « une place nouvelle» (Auster, 1975 : 61 et 54). De même, pour Le Clézio, « chaque fois qu’un de ces livres paraît, [...] alors il semble que c'est la littérature tout entière qui soit remise en question » (Le Clézio, $1970: 39)$.

Ces observations ont en commun de substituer à la valeur littéraire conventionnelle une valeur d'exception : l'œuvre ne peut être admise dans la littérature qu'à condition d'en changer la définition. «Livre exceptionnel », « objet admirable », et donc du même coup objet à part, hors normes, Le Schizo et les langues instruit la littérature sans nécessairement en faire partie. Par ce biais le livre demeure « sécurisé » » comme objet à part.

Une semblable éviction de la sphère littéraire s'observe dans le mouvement américain de la « schizo culture ». Le terme "schizo » connaît alors un très large écho, comme en témoigne le colloque « Schizo culture » organisé en 1975 par la revue Semiotext(e). La « schizo-culture » est conçue comme « a revolution in desire », et le "schizo » devient un terme désignant la lutte à l'oppression, incarnant un rapport différent à la vérité. Aux côtés de Deleuze et Guattari, William Burroughs et d'autres grands noms de la french theory, Sylvère Lotringer invite Wolfson à parler, mais ce dernier se désiste en fin de compte (Lotringer, 2013). Le texte du «jeune homme schizophrénique », publié dans le numéro suivant le colloque (Wolfson, 1975), se trouve alors noyé dans une sphère ontologique, qui élude son discours et la portée qu'il lui donne.

Pourquoi Le Schizo et les langues a-t-il difficilement été envisagé comme un texte littéraire? Wolfson se montrait peut-être trop lucide sur sa propre condition de "schizophrène », revendiquant trop l'étiquette qu'on lui attribuait. Wolfson joue de lui-même comme un type, un stéréotype, à l'aide des périphrases («le jeune homme schizophrénique »), de l'usage froid et distancé de la troisième personne. Il lui manque le « beau délire » que le public, selon toute une tradition qui remonte à L'Éloge de la folie en passant par Une saison en enfer, attend des discours fous pour les accepter dans la littérature.

Les années 1970 ont eu comme mérite de remettre en perspective les discours hors-normes des psychotiques, de les interroger comme discours et de sortir ces réflexions du domaine uniquement psychiatrique ou psychanalytique. Ce qui s'exprime est donc la possibilité que la folie instruise la littérature. C'est ce qu'on trouve, au-delà de l'avant-garde, chez Deleuze et Guattari :

Se dessine la vision d'un schizophrène «branché » sur les flux du désir que la société refrène, véritable connaisseur de la folie comme force supérieure et détenteur du langage schizophrénique dont on peut espérer l'accomplissement de la littérature. (Deleuze, 1972: 160)

Mais nous sommes forcée de constater l'échec de cette prophétie. Le discours psychotique n'a jamais permis l'accomplissement de la littérature, tout au plus a-t-il donné ses lauriers à la littérature en demeurant en retrait. La construction d'une sorte de mythe du fou considéré comme contestataire, porteur de révolution en soi, a souvent conduit à confondre le message et la personne qui en était le vecteur. L'étude de la représentation de ces discours hors-normes dans l'avant-garde littéraire nous a ainsi permis de montrer que le discours « fou » vaut davantage comme symbole que par son réel contenu linguistique à l'image d'une «schizo-culture » qui s'est en réalité éloignée des malades mentaux eux-mêmes. Le discours fou éclipse le discours des fous. 


\section{Bibliographie}

Abrahams Jean-Jacques (1976), «L’homme au magnétophone (suites) », Tel Quel, 65, 35-40.

Abrahams Jean-Jacques (1976), «Intervention », Tel Quel, 67, 102-103.

Althusser Louis (1970), «Idéologie et appareils idéologiques d’État. Notes pour une recherche » La Pensée, 151, juin 1970, repris dans Positions (1964-1975), Paris, Les Éditions sociales, 1976, 67125.

Armelino Michel et Buin Yves (1975), «Formes de l'expérience », Collectif Change, Change matériel, t. II, Folie, histoire, récit, Paris, 10/18, 15-61.

Auster Paul (1975), « New York Babel », J.-B. Pontalis et alli., Dossier Wolfson, ou L'affaire du Schizo et les langues, Paris, Gallimard, « L’Arbalète », 2009.

Balibar Renée (1974a), Les Français fictifs, le rapport des styles littéraires au français national, Hachette Littérature, coll. «Analyse ».

Balibar Renée et Laporte Dominique (1974b), Le Français national, politique et pratique de la langue nationale sous la révolution, Hachette Littérature, coll. «Analyse».

Baudry Jean-Louis (1968), «Écriture, fiction, idéologie », dans Théorie d'ensemble, Paris, Seuil, coll. « Tel Quel », 127-147.

Buin Yves (1972), «Sur la fonction du traître (Journal de Sarreguemines) », Change, 12, Déraison désir.

Breton André (1959), Perspective cavalière, Paris, Pléiade, t. IV, 981.

Delahaye Yves (1976), «Pour une sémiotique des relations internationales », Tel Quel, 67, p. 63-75.

Deleuze Gilles (1970), « Schizologie », préface au Schizo et les langues, Paris, Gallimard, 5-23.

Deleuze Gilles et Guattari Félix (1972), L’Anti-CEdipe, Paris, Les Éditions de Minuit, coll. « Critique ».

Dosse François (1992), Histoire du structuralisme, t. II, Le Chant du cygne, Paris, La Découverte.

Faye Jean-Pierre (1974), «Le mouvement du change des formes », Change, 18, Mouvement du change des formes, 5-20.

Faye Jean-Pierre (dir.) (1975), Change, 24 « Mouvement du change de forme et transformationnisme ».

Houdebine Jean-Louis (1968), «Première approche de la notion de texte », dans P. Sollers et alii., Théorie d'ensemble, Paris, Seuil, coll. «Tel quel », 270-284.

Joseph John E. (2011), «Théories et politiques de Noam Chomsky », Langages 2011/2, 182, 55-67.

Kaufmann Vincent (2011), La Faute à Mallarmé, l'aventure de la théorie littéraire, Paris, Seuil, coll. « La couleur des idées ».

Kristeva Julia (1969), « Le mot, le dialogue, le roman », dans Semeiotikè, recherches pour une sémanalyse, Paris, Seuil, coll. «Points Essais », 1978.

Kristeva Julia (1974), La Révolution du langage poétique. L'avant-garde à la fin du XIXe siècle, Paris, Seuil, coll. «Tel Quel ».

Kristeva Julia (1977), « Pourquoi les États-Unis », Tel Quel, 71-73. 
Le Clézio J. M. G. (1970), « La tour de Babil », repris dans Jean-Bertrand Pontalis et alii., Dossier Wolfson, ou L'affaire du Schizo et les langues, Paris, Gallimard, « L'Arbalète », 2009.

Leclaire Serge (1972), «Les mots du psychotique », Change, 12, Déraison désir.

Semiotext(e) (1975), colloque « Schizo culture », 13-16 novembre 1975, Colombia University, S. Lotringer et D. Morris (dir.), Schizo-culture, The Event, 1975, Los Angeles, Cambridge (Mass.) et Londres, Semiotext(e)/ The MIT Press, 2013.

Milner Judith (1977), "Frontières de langue : de quoi rient les locuteurs? » (II), Suivi de « Langue et folie », Change, 32-33, La folie encerclée, 131-162.

Moreau-Hicks Thérèse (1973), «Dendrites », Change, 16-17, La Critique générative, repris dans Change matériel, t. II, «Folie, histoire, récit », 1975.

Pleynet Marcelin (1973), Stanze, Paris, Seuil, coll. « Tel Quel».

Podolski Sophie (1973), « Le pays où tout est permis », Tel Quel, 53, 5-16.

Podolski Sophie (1973b), «Et toujours... », Tel Quel, 55, 94-97.

Podolski Sophie (1977), « Fragments inédits », Tel Quel, 74, 78-83.

Rey Alain (1970), « Le Schizolexe », Critique, 279-80, 677-691.

Roche Denis (1978), «L'esprit fou dans les grands vents », TXT, 10, L'ÉcriT, le CacA, 65-68.

Ronat Mitsou (1975), " Note sur L'Inconscient des langues », Change, 24, Mouvement du change de forme et transformationnisme, 173-175.

Ronat Mitsou (1976a), « Mallarmé : visible syntaxe », Change, 26-27, 171-174.

Ronat Mitsou (1976b), «Rythme et syntaxe en prose mallarméenne (visible syntaxe II) », Change, 29, Le sentiment de la langue, 19-37.

Ronat Mitsou (1976c), «Les impressionnistes et Edouard Manet », traduction de Mallarmé, Change, 29, Le sentiment de la langue, 58-75.

Roudinesco Elisabeth (1970), «L'inconscient et ses lettres », Action poétique, 45, 46-68.

Ruwet Nicolas (1967), Introduction à la grammaire générative, Paris, Plon.

[s. a.] (1975) « Manifeste du change en d'autres termes, Manifesto di "Change" in "Altri termini”, J.-P. Faye (dir.), (1975) Change, 24,Mouvement du change de forme et transformationnisme.

Sartre Jean-Paul (1969), «L'homme au magnétophone », Les Temps Modernes, 274, avril 1969, repris dans Situations, IX, mélanges, Paris, Gallimard, 1972, 329-337.

Sabourin Danielle et Polack Jean-Claude (1975), « Le langage du grand groupe », dans Collectif Change, Change matériel : changement de forme, révolution, langage, Paris, 10/18, 1975.

Sibony Daniel (1977), «Écriture et folie », Tel Quel, 70, 46-60.

Sollers Philippe (1968a), Logiques, Paris, Seuil.

Sollers Philippe (1968b), L'Écriture et l'expérience des limites, Paris, Seuil. 
Sollers Philippe (1972), «L'état Artaud », Tel Quel, 52, 3-11.

Sollers Philippe (1975), «La renommée», Tel Quel, 64, 100-101.

Sollers Philippe (1977a), «Deux interventions aux Etats-Unis », Tel Quel, 69, p. 6-10.

Sollers Philippe (1977b), «"Folie”, mère-écran », Tel Quel, 69, 97-102.

Starobinski Jean (1964), « Les anagrammes de Ferdinand de Saussure », Le Mercure de France, février 1964, n 6, 243-262.

Starobinski Jean (1969), «Le texte dans le texte, extraits inédits des cahiers d’anagrammes de Ferdinand de Saussure », Tel Quel, 37, 3-33.

Starobinski Jean (1971), Les Mots sous les mots. Les anagrammes de Ferdinand de Saussure, Paris, Gallimard, coll. «Le chemin».

Thevoz Michel (1978), « Extraits des carnets de Jules Doudin » suivi de « Les carnets de Jules Doudin », TXT, $n^{\circ} 10$, l'ÉcRlt, le CacA, 15-20.

Wolfson Louis (1970), Le Schizo et les langues, Paris, Gallimard, coll. «Connaissance de l'inconscient ».

Wolfson Louis (1977), «L'épileptique sensoriel schizophrène », extraits remaniés du Schizo et les langues, Change, 32-33, octobre 1977, et, mars 1978, 34-35.

Wolfson Louis (1975), « Full Stop for an Infernal Planet or The Schizophrenic Sensorial Epileptic and Foreign Languages », Semiotext(e), vol. 3, n’ 2, rééd. 2013, Semiotext(e), The Book, Los Angeles, Cambridge (Mass.) et Londres, Semiotext(e)/The MIT Press, 44-46.

Zoïla Adolfo Fernandez (1975), «Edmond Jabès et les structures éclatées », Change, n²2, L'imprononçable, l'écriture nomade. 